\title{
Image Super-Resolution Reconstruction Using Adaptive Co-sparse Regularization with Dual Dictionary
}

\author{
Muhammad Sameer Sheikh, Qunsheng Cao, and Caiyun Wang
}

\begin{abstract}
This paper present a new method based on co-sparse with learning paired dictionary. The new framework is consisted of three parts. Firstly a paired dictionary have been learned which is used to overcome a low resolution image by utilizing an externally applied high resolution (HR) dictionary and then learn based on the internal dictionary. Process the paired dictionary which consists of low resolution (LR) and high resolution (HR) dictionary by kernel regression based on their coefficient respectively, and applied directly to construct the HR patches. Secondly, co-sparse regularization and features of self similarity have been introduced to strengthen and enhanced the image structure. In addition, propagation filtering is applied to suppress the artefacts generated from neighboring pixel of an image while reserving the image edges. Finally, the HR image is generated by reconstructing all superior HR patches. The effectiveness of the co-sparse demonstrated in real test images. The proposed method achieved good quality high resolution images that are superior compared with different $S R$ methods in terms of peak signal to noise ratio (PSNR), and structural similarity (SSIM).
\end{abstract}

Index Terms-Dual dictionary, image resolution, propagation filter, self-similarity.

\section{INTRODUCTION}

Image super-resolution reconstruction is very vital and active research field because it inverses the limitations of resolution in digital imaging. SR is acts as a process of reconstructing a high resolution (HR) image from low resolution (LR) input images, and applied successfully in many application such as, satellite imaging, biomedical imaging and electronic imaging etc. In recent years, SR reconstruction imaging model receives a great deal of attention [1], and several SR approaches have been proposed to overcome the inherent limitation of resolution.

SR method can be divided into three categories, such as interpolation-based methods, reconstruction-based methods, and learning-based methods respectively. The interpolation method such as bicubic method, this method is very simple and fast but can generates blurred artefacts in the reconstruction part of SR image [2]. To inverse this effect, several methods have been proposed based on edge-direction interpolation method to preserve the edges. Due to limitation

Manuscript received June 23, 2016; revised August 15, 2016. This work is supported by the National Natural Science Foundation of China (no. 61301211) and the Key Laboratory of Radar Imaging and Microwave Photonics, Ministry of Education, Nanjing University of Aeronautics and Astronautics, Nanjing, 210016, People Republic of China.

Muhammad Sameer Sheikh and Qunsheng Cao are with the College of Electronics and Information Engineering, Nanjing University of Aeronautics and Astronautics, Nanjing, China (e-mail: sameer.5@hotmail.com).

Caiyun Wang is with the College of Astronautics, Nanjing University of Aeronautics and Astronautics, Nanjing, China. of modeling the rich detailed of natural images, therefore, it is unable to generate the SR image [3], [4]. Reconstruction-based method SR methods need certain input information to construct the HR image. It usually assumes the LR input image which is the combination of several factors such as blurring and down sampling operator, and noise. In this method one LR image relates to several HR images which leads to an ill posed problem. Smooth edge technique has been introduced to preserve the edges of image and strengthen the image structure [5]. Several methods have been proposed which named as iterative back projection [6], [7], and maximum a posteriori (MAP) [8], [9]. Firstly it decomposes the original image into low pass and band pass, sub band images by using non-sampled transform. Secondly it applies edge detection method to reconstruct the SR image with good edge preservation. These approaches yielding good reconstruction quality of SR image but can generate aliasing effect in the reconstruction part.

Lately, the learning-based method have been very popular and play a major development in digital imaging. The example based method patch are modeled using Markov network and each element in the HR image comes from neighbor sets in the training phases [10], this method generates good SR image with smooth edges and better resolution but requires large computational time to process of neighboring patches. Learning framework for example based method has been proposed recently, which fully consumes the HR distribution in terms of LR patches via soft information and decision [11]. Chakrabati et al. [12] proposed a method to reconstruct an SR image using kernel principal component analysis method (PCA), but this approach is limited to face images [12]. Kim and kwon [13] introduced a new method to reconstruct an SR image based on regression, the approach has been done based on study conducted by Freeman et al. This method can generate good HR image while maintain the edge preserving but requires large computational time. Learning machine technique has been used to generate weighting matrix of every LR image this method can produce good SR image but consume more time to generate an SR image [14].

Sparse method has been widely used in digital imaging and pattern recognition, based on this concept several methods have been proposed, such as dictionary learning based method which provides robust SR reconstruction and satisfactory reconstructed SR image. Yang et al. [15], [16] used sparse coding to reconstruct an SR image based on joint learning dictionary by assuming the concept that natural images have similar image structure and high frequency HF component. This method provides SR image with jaggy artefacts because of missing high frequency information in the reconstruction part. Yu et al. [17] introduced a modeling 
pursuit method. Firstly, it computes the image decomposition over spaces and then generates an HR image. Zeyde et al. [18] proposed a new training method for paired dictionary which is used to reconstruct an SR image, and algorithm is still time consuming because of learning paired dictionary. Yincheng et al. [19] proposed a robust method to reconstruct the HR image based on redundant dictionary, but it required large amount of calculation to process the dictionary under training phase. Dong et al. [20] has introduced the adaptively sparse regularization technique which utilizes a dictionary learning model which is constructed from a high resolution patches to generate an SR image, this approach inverses the artefacts and produce good resolution image with rich texture but it consumes large memory. New method has been applied recently to achieve the goal of an SR image by externally applied high resolution dictionary to reconstruct an image, the applied dictionary is not selected adaptively and processing of each patch required large amount of calculation, consequently leads to be large time consuming algorithm [21]. Chen et al. [22] introduced an adaptive cosparse method to reconstruct a high resolution HR image, patch is divided under the subspace domain by cluster form and then learned the subdictionary from cluster respectively, and the resulting SR image is with less texture detailed due to missing HF component in applied dictionary. Recently, SR can be reconstruct based on clustering algorithm by using sparse algorithm, the dictionary is used in reconstruction phase which is unable to produce a satisfactory SR reconstruction [23].

From the above discussion several methods achieve the goal of SR reconstructions, but there are still some limitations and some detailed information are lost, when reconstructing from LR input image to HR image. In this study, we proposed a new method which uses co-sparse algorithm with paired dictionary to reconstruct an SR image, referring to the same idea [21]. The proposed method utilizes the prior input information which is get from dictionary of internal LR image, and external HR image respectively, to achieve the better resolution HR image. The co-sparse regularization and self-similarity terms have been introduced to strengthen and smooth the edges. Finally the propagation filter is used to suppress the artefacts. It can be seen that the proposed method can generate better SR reconstruction.

\section{Problem Discussion In SR Image}

Fig. 1 shows the framework of the proposed method, which achieves the goal of SR image by utilizing the external dictionary. Inspired from the idea of [21], we have used two dictionaries to achieve the most part of the input LR image. Firstly, we construct an internal LR dictionary with an input LR image. Secondly, an externally applied dictionary is constructed from a high resolution images which is select based on clustering and then, compute the structure of clustering by using LR patches to gain the high frequency (HF) component respectively. In addition, developing the relationship between the LR and HR coefficient by using kernel regression method to obtain the smooth image. The final HR image is constructed by reconstructing all HR patches.

Generally the reconstruction of HR image can be developed from LR input images but resolution and brightness remains critical issues. The main problem exists when reconstruction of HR image from LR input image cause severely ill-posed problem because of less sufficient information in LR images. Let assume $Y_{l}$ and $X_{h}$ are the input LR and HR image.

$$
Y_{l}=L B X_{h}+n
$$

where $n$ is the Gaussian noise, and $L$ and $B$ are the down sampling and blurring operators respectively. The estimated HR image satisfying the reconstruction constraint with input LR image as shown below,

$$
X_{h}=\arg \min \quad x_{h}\left\|Y_{l}-L B X_{h}\right\|_{2}^{2}
$$

The above (2) is the image restoration model, which is used to produce an SR image based on the low resolution input image.

\section{A. Paired Dictionary Learning}

To learn a dictionary and to strengthen the image structure, we need to obtain a dataset of image patches for training of an image. To achieve this we gathered a HR images and cropped the image which contain rich information with size of $\sqrt{n} \times$ $\sqrt{n}$. The image is divided into two categories such as smooth and texture. Let assume the $m^{\text {th }}$ input image is $y_{h}^{m}$ and it is scaled up by using the interpolation to obtain the $\mathrm{n}$ images which has the equivalent size of original image $x_{h}$. Then extract the high frequency component of $n$ images which LR image may lost. Firstly, image characteristics is further categorized. Let assume input image $P_{i}=\left\{P_{1}, P_{2}, \ldots, P_{n}\right\}=$ $\left\{P_{i}\right\}_{i=1}^{n}$ and the equivalent original image is $Q_{i}=$ $\left\{Q_{1}, Q_{2}, \ldots, Q_{n}\right\}=\left\{Q_{i}\right\}_{i=1}^{n}$. We cropped a rich amount of image patches from $Q_{i}$ and $P_{i}$ with the size of $\sqrt{n} \times \sqrt{n}$. It can be represented as a patch sets of $P_{t}$ and $\mathrm{Q}_{\mathrm{t}}$ respectively. After successfully creating the $P_{t}$ dataset we are now clustering $P_{t}$ datasets into $k$ cluster which is $\left[C_{1}, C_{2}, \ldots, C_{K}\right]$. The patches are passed through high pass filter to form a structure of the image patches and edges, $P_{t}^{h}=\left[P_{1}^{h}, P_{2}^{h}, \ldots \ldots, P_{n}^{h}\right]$ so $P_{t}^{h}$ can be written as $P_{t}$ and the dataset $Q_{t}$ can be clustered into subsets $Q_{t}=\left\{Q_{t 1}, Q_{t 2}, \ldots, Q_{t k}\right\}$, and $Q_{t k}$ be the $k^{t h}$ subset. Similarly the same clustering is performed on the high resolution HR image patch selection, and we can achieved a patch sets of $\left\{P_{t}, \dot{Q}_{t}\right\}$, and $Q_{t}$ is clustered into $k$ subsets so $Q_{t}=\left\{Q_{t 1}^{\prime}, Q_{t 2}^{\prime}, \ldots, Q_{t k}^{\prime}\right\}$, and $Q_{t k}^{\prime}$ be the $k^{t h}$ subset.

\section{B. Principal Component Analysis (PCA)}

Principal component analysis (PCA) is powerful technique which is used as a linear transformation based on statistical technique. PCA plays a vital role in digital signal processing, and is mainly used as a dimension reduction technique, and de-correlation [24]. This method has been successfully applied in image denoising by applying on each image patch [25], and can be used to find out the orthonormal transformation matrix. By applying the PCA to each datasets $Q_{t}$ and $Q_{t}$ from which the dictionaries is constructed therefore 
we achieves the $K$ internally $d j$ and $K$ externally $\mathrm{Di}$ dictionaries respectively. After successfully applying PCA, we get the eigenvectors which then form a subdictionary Dk. let assume $D_{k}=\left[q_{1}, q_{2}, \ldots, q_{e}\right]$, so we can written as,

$$
\mathrm{s}=\arg \min _{\mathrm{s}}\left\|S_{k}-D_{k} \mu_{\mathrm{k}}\right\|_{F}^{2}+\rho\left\|\mu_{\mathrm{k}}\right\|_{1}
$$

where $\mu_{k}=D_{k}^{T} S_{k}$ is the sparse representing coefficients of $\mathrm{S}_{\mathrm{k}}$. $D_{K}$ is the subdictionary of the $k^{\text {th }}$ cluster.

\section{KeRNEL REGRESSION METHOD}

Specifically the down sampling method is the process to transform an HR image into LR image which can be categorizes as a linear process, and to reproduce the HR image by using its corresponding LR image can be act as an ill-posed problem. To overcome this problem we are cluster the HR and LR image patches into K clusters, and then train a series of mapping function [26].

We are now developing the relationship between the LR and HR coefficients. To achieve this, we are categorizing HR LR image patches into $\mathrm{K}$ clusters, and train a mapping functions.

$$
\begin{aligned}
& \alpha_{j k}=d_{j k} S_{j k}=\left[\gamma_{j, 1, \ldots,}, \gamma_{j, n}\right] \\
& \alpha_{i k}=D_{i k} S_{i k}=\left[\gamma_{i, 1, \ldots,}, \gamma_{i, n}\right]
\end{aligned}
$$

where $\gamma_{j,\{z\}_{z}=1, \ldots n} \in \mathrm{R}^{N_{j} \times 1}$ and $\gamma_{i,\{z\}_{z}=1, \ldots n} \in \mathrm{R}^{N_{i} \times 1}$ and $S_{j k}$ and $S_{i k}$ are the training LR and HR patches related to $\mathrm{k}$ clusters. $d_{j k}$ and $D_{i k}$ are the LR and HR dictionaries, and $\alpha_{j k}$, $\alpha_{i k}$ are the coding coefficients along with the LR and HR dictionaries respectively. Sectioned into input and output values which contain $n$ number of pairs so training set can be written as below

$$
\emptyset=\left\{\left(\gamma_{j, 1}, \gamma_{i, 1}^{t}\right), \ldots,\left(\gamma_{j, 2}, \gamma_{i, 2}^{t}\right), \ldots,\left(\gamma_{j, n}, \gamma_{i, n}^{t}\right)\right\}
$$

As discussed earlier $\gamma_{j, z}$ is the input vector, and $\gamma_{i, z}$ is the output vector respectively. At last passed the training sets through the kernel

$$
K\left(\gamma_{j}\right)=\sum_{z=1}^{n}\left(\beta_{z}-\dot{\beta}_{z}\right) k\left(\gamma_{j, 1}, \gamma_{j, z}\right)+C
$$

The parameter $k$ is responsible of HR patch coefficient $\gamma_{i}$ and contained all values of the cluster. After successfully trained $k$ which can formed the relationship between LR and HR coefficient, where $\beta_{z}$ and $\dot{\beta}_{z}$ are the langrange multiplier [26].

\section{Co-Sparse SignAl AnAlysis MethoD}

Generally, in learning based method we used input LR image to achieve the goal of SR image. The LR image is blurred with noise effect. Recently several methods have been proposed to inverse this effect. Co-sparse method provides robust and unique approach of the linear problem in digital image processing model. Inspired from the basic concept of co-sparse analysis [27]. In sparse by using the operator function sometime image is not sparse, but its gradient can sparse [28].

$$
X_{h}=\arg \min _{x_{i}}\left\|Y_{l}-L B X_{h}\right\|_{2}^{2}+\mu\left\|\sum_{i=1}^{N} D_{k} x_{i}\right\|_{0}
$$

where $\mu$ is the regularization coefficient to maintain the balance of co-sparsity.

Eq. 8 represent the co-sparse signal, $D_{k} x_{i}$ represents the feature from $x_{i}$, and $D_{k}$ is the subdictionary with size of $D_{k} \in R^{p \times n}$, and \|\|$_{0}$ is the 10 pseudo-norm.

\section{A. Self-Similarity Features}

To reconstruct a better SR image, we introduce the nonlocal self-similarity features which will improved the reconstruction quality. In the full image which contains similar patch or patterns. We rely on the same idea of [20]. Each patch $x_{i}$ has similar patches with in the whole image $\mathrm{x}$, let assume $\boldsymbol{x}_{\boldsymbol{i}}$ be the center pixel of the patch $x_{i}$ and $\boldsymbol{x}_{i}^{l}$ is the center pixel of patch of $x_{i}^{l}$, we can estimate the patch $x_{i}$ by using weighted average of $\boldsymbol{x}_{\boldsymbol{i}}^{l}$, which can be written below,

$$
x_{i}^{l}=b_{i}^{l} x_{i}^{l}
$$

where $b_{i}^{l}$ is weight assigned to the $x_{i}^{l}$.

Natural images contains some patch redundancy. By calculating the error which has been updated $e=$ $\left\|x_{i}-\boldsymbol{x}_{\boldsymbol{i}}^{\boldsymbol{l}}\right\|_{2}^{2}$. Incorporate the nonlocal similarity term into the co-sparse based representation method to enhance the performance of the sparse signal so Eq. (8) can be written as below,

$$
\begin{gathered}
X_{h}=\arg \min _{x_{i}}\left\|Y_{l}-L B X_{h}\right\|_{2}^{2}+\mu\left\|\sum_{i=1}^{N} D_{k} x_{i}\right\|_{0}+ \\
q \cdot\left\|x_{i}-x_{i}^{l}\right\|_{2}^{2}
\end{gathered}
$$

where $q$ is the constant parameter which balance the nonlocal similarity term and suppresses the blurred artefacts and also enhances the resolution of reconstructed image with presence of similar patches.

\section{B. Propagation Filtering}

Propagation filter has been successfully applied in several imaging application such as image denoising, image fusion and, image compression techniques. We are now utilizing the propagation filter [29]. This method achieves the goal of smoothing with neighbor pixels while maintaining the image structure and preserving edges.

$$
\begin{array}{r}
X_{h}=\arg \min _{x_{i}}\left\|Y_{l}-L B X_{h}\right\|_{2}^{2}+\mu\left\|\sum_{i=1}^{N} D_{k} x_{i}\right\|_{0}+ \\
q \cdot\left\|x_{i}-\boldsymbol{x}_{\boldsymbol{i}}^{l}\right\|_{2}^{2}+m\left\|X-X_{f}\right\|_{2}^{2}
\end{array}
$$


where $m$ is a regularization coefficient, and $X_{f}$ is the filter output.

The filter based regularization which can maintain the smooth edges of the reconstructed HR image, but may suppress the rich detailed information of the HR image. Therefore selection of regularization parameter $m$ is very important issue to obtain the optimum solution required for reconstruction of HR image. In (11) the first term guarantee the recovering $X_{h}$ from the degraded input LR image $Y_{l}$, and second term used to enhances the image structure, the third term is the nonlocal self-similarity, and fourth term is propagation filter which maintain the edge structure of reconstructed HR image.

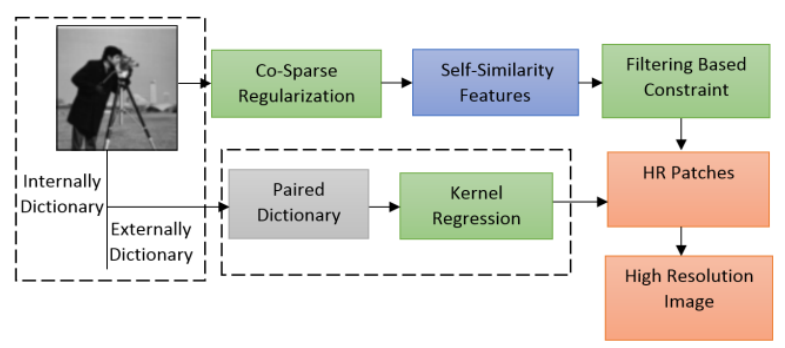

Fig. 1. Block diagram of proposed model.

\section{SR IMAGE RECONSTRUCTION}

In SR imaging method, the HR image can be reconstructed by LR image based on co-sparse signal with subdictionary. Let assume the LR patch $y_{l}$, and the internal subdictionary along with cluster is $d_{j k}$, so we achieved sparse signal of $y_{l}$ i.e. $\gamma_{j}=d_{j k}^{T} y_{l}$.

$$
\gamma_{i}=\left[f_{1}\left(\gamma_{j}\right), \ldots \ldots, f_{n}\left(\gamma_{j}\right)\right]
$$

Specifically, the HR patch can be recovered. The low resolution input image $Y_{l i}$ is used to scale up by using the bicubic method to achieve the corresponding high resolution image $X_{\mathrm{h}}$ and have the same size as $X_{i 1}$. Let assume $X_{\mathrm{h}}$ is the high resolution image vector, and $Y_{l i}$ is the low resolution image vector, then feature of image $Y$ can be defined as, $Y=R_{k i} Y_{l i}$ where $\mathrm{i}$ is the number of patches, $\mathrm{i}=1,2,3 \ldots \mathrm{N}$, and $R_{k i}$ is the matrix extraction feature of patch $Y$ from $Y_{l i}$.

$$
P_{h}=\sum_{k=1}^{K} \sum_{i=1}^{N} R_{k i} Y_{l i}
$$

In Eq. 13 the term $R_{k i} Y_{l i}$ which builds the high resolution patches.

$$
\begin{array}{r}
X_{h}=\arg \min _{x_{i}}\left\|P_{h}-L B X_{h}\right\|_{2}^{2}+\mu\left\|\sum_{i=1}^{N} D_{k} x_{i}\right\|_{0}+ \\
q \cdot\left\|x_{i}-x_{i}^{l}\right\|_{2}^{2}+m\left\|X-X_{f}\right\|_{2}^{2}
\end{array}
$$

Our objective is to generate the HR patches, which can be possible by learning a subdictionary and co-sparsing coefficient.

$$
\operatorname{argmin}_{X_{h}}\left\|\left(R_{k i} Y_{l i}\right)-P_{h}\right\|_{2}^{2}
$$

$$
X_{h}=X_{i 1}+P_{h} R_{i}^{T} R_{i}
$$

The final HR image can be constructed by averaging all reconstructed patches $P_{h}$ in overlap region and adding $X_{i 1}$ to achieve the final image which contain rich detailed information. Where $R_{i}^{T} R_{i}$ is a diagonal matrix which weights the each pixels of high resolution image.

\section{EXPERIMENTAL RESULTS}

In this section, to evaluate the performance of the proposed method several experiments have been conducted to comprehensively demostrate the effectiveness of the proposed method. The LR image is downsampled from HR image with the scaling factor of 3 , and the patch size of $7 \times 7$ has been used.

\section{A. Patch Size Evaluation}

In this subsection, to evaluate the visual quality of the proposed method. We are carrying out SR experiment on cameraman image to compare the visual quality with different patch sizes $(3 \times 3),(5 \times 5)$, and $(7 \times 7)$ respectively.

To evaluate the visual comparison by using different patch sizes by implementing on cameraman image. Patch size of $(3 \times 3)$ and $(5 \times 5)$ may not produce the robust reconstruction of HR image because of utilization of limited number of pixels which can also generate blurred artefacts. In proposed method we have chosen the patch size of $(7 \times 7)$, which can produce better reconstruction of HR image with good contrast and resolution, and also suppresses blurred artefacts.

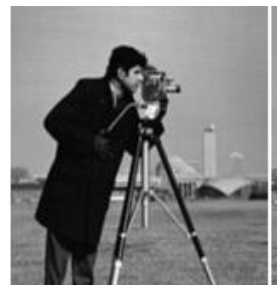

(a) $3 \times 3$

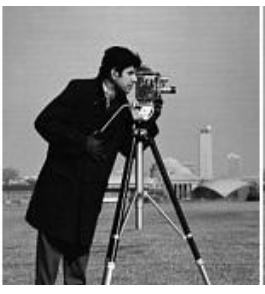

(b) $5 \times 5$

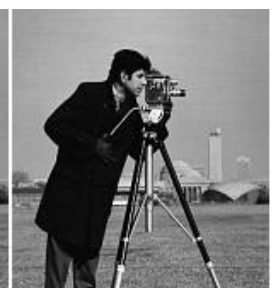

(c) $7 \times 7$
Fig. 2. Patch comparison.

\section{B. Effectiveness of Dual Dictionary and Propagation Filtering}

As we know that each pair of high resolution and low resolution image patches have contains the same co-sparse representation of signal which is based on internal $d_{j}$ and external $D_{i}$ dictionary respectively. However, by processing internal dictionary and directly applied the external dictionary would produce the reliable solution while maintaining the computational time. Firstly, we have trained HR dictionaries from the high resolution images and applied externally to achieve the good resolution of super resolution HR image.

Propagation filter can be widely applied in many imaging techniques, such as image denoising, image smoothing, image fusion etc. To suppress the large amount of unwanted information from neighboring pixels, we successfully apply propagation filter which is used to achieve the smoothing goal over neighboring pixels while maintaining image structure of reconstructed HR image. The selection of $m$ 
value is critical issue, if we select small $m$ value it cannot preserve the image, and may loss the rich detailed information. Indeed by larger value of $m$ we can achieved the image smoothing structure while maintaining image edges, but that can generate little sharp edges.

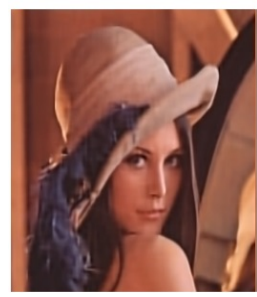

(a) HR image

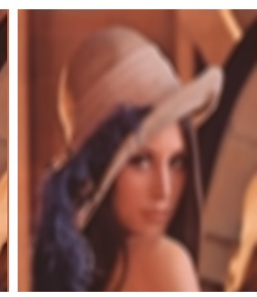

(b) Recursive filter output

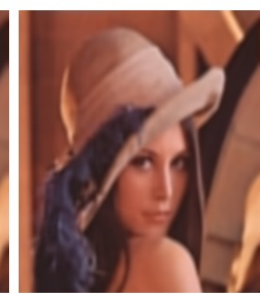

(c) Propagation filter
Fig. 3. Effect of propagation filter.

In Fig. 3, we are now comparing the image smoothing, and analysis of image structure of propagation filter with recursive filter by using Lena image. Fig. 3(a) shows the original HR image, Fig. 3(b)-(c) show the recursive filter output and propagation filter output respectively. It is shown that Fig. 3(b) cannot preserve the image edges due to overlapping of neighboring pixel and cannot produce smoothing image. In our method we select the optimum value of $m$ which leads to be smoothing image, while preserving the image edges with rich detailed information

\section{Experiment with Different Images}

In this section, several experiments have been conducted to demonstrate the qualitative and quantitative effectiveness of the proposed method by implementing on seven different images (house, peppers, baboon, cameraman, straw, lena, and flower). Fig. 4(a) shows the original HR image, 4(b) shows the bicubic interpolation method, and 4(c)-(e) show the results of various algorithm Chen [22], Gou method [21], and the proposed method respectively. It can be seen from Fig. 4(b), that the bicubic method results contained jaggy artefacts and produce degraded SR image, and are unable to overcome the deblurring problem. Chen [22], overcome the limitation of bicubic method by providing better algorithm which leads to be satisfactory SR reconstruction that contained a better visual quality but having less texture detailed compare with proposed method. Gou [21] achieved better results and outperforms previous method in terms of visual resolution but cannot maintain the smoothing edges of reconstructed SR image. In Fig. 4(e), the proposed method can generate better SR image with enhanced resolution and smoothing edges, and that also outperforms several competent methods.

\section{Comparison with PSNR}

In this subsection, we are analyzing the peak signal to noise ratio (PSNR) comparison of the proposed method with other competent method by using seven images see Table I. with $\sigma=0$. It can be seen that bicubic method achieved low PSNR in all of applied images. Chen [22] algorithm can generated SR image and achieved better PSNR results than the bicubic method. Gou [22] approach achieved better PSNR because of applied external dictionary which is constructed from high resolution images and that leads to be good reconstructed SR image. The proposed method significantly improves the quality of the reconstructed SR image with higher PSNR values in all of the images and also outperforms several other methods.

\section{E. Comparison with SSIM}

The evaluation results of SSIM of different methods are shown in Table II. with $\sigma=0$. SSIM values varies from different images. In can be seen that the bicubic method has less sufficient details which leads to unpleasing reconstructed image, therefore this approach achieves less SSIM values in all images. In addition, we can see that the proposed method achieves slightly higher SSIM values compared with other methods, and which also outperforms competent method such as Bicubic method, Chen [22], and Gou [21].

TABLE I: RESULT OF PSNR COMPARISON FOR DIFFERENT IMAGES

\begin{tabular}{|c|c|c|c|c|}
\hline Image & $\begin{array}{c}\text { Bicubic } \\
\text { method }\end{array}$ & Chen [22] & Gou [21] & $\begin{array}{c}\text { Proposed } \\
\text { Method }\end{array}$ \\
\hline House & 27.61 & 28.52 & 29.29 & 29.35 \\
\hline Peppers & 23.56 & 25.81 & 26.75 & 27.48 \\
\hline Baboon & 21.85 & 22.26 & 23.89 & 25.64 \\
\hline Cameraman & 27.05 & 28.35 & 28.69 & 29.22 \\
\hline Straw & 21.98 & 22.68 & 23.74 & 24.65 \\
\hline Lena & 24.31 & 24.95 & 26.15 & 27.58 \\
\hline Flower & 27.32 & 29.15 & 29.36 & 30.15 \\
\hline
\end{tabular}

TABLE II: RESULT OF SSIM COMPARISON FOR DIFFERENT IMAGES

\begin{tabular}{|c|c|c|c|c|}
\hline Image & $\begin{array}{c}\text { Bicubic } \\
\text { method }\end{array}$ & Chen [22] & Gou [21] & $\begin{array}{c}\text { Proposed } \\
\text { Method }\end{array}$ \\
\hline House & 0.7860 & 0.8150 & 0.8305 & 0.8412 \\
\hline Peppers & 0.8510 & 0.8625 & 0.8712 & 0.8864 \\
\hline Baboon & 0.8051 & 0.8125 & 0.8365 & 0.8455 \\
\hline Cameraman & 0.8515 & 0.8612 & 0.8755 & 0.8835 \\
\hline Straw & 0.7365 & 0.7415 & 0.7512 & 0.7615 \\
\hline Lena & 0.8541 & 0.8601 & 0.8715 & 0.8816 \\
\hline Flower & 0.8215 & 0.8481 & 0.8553 & 0.8612 \\
\hline
\end{tabular}




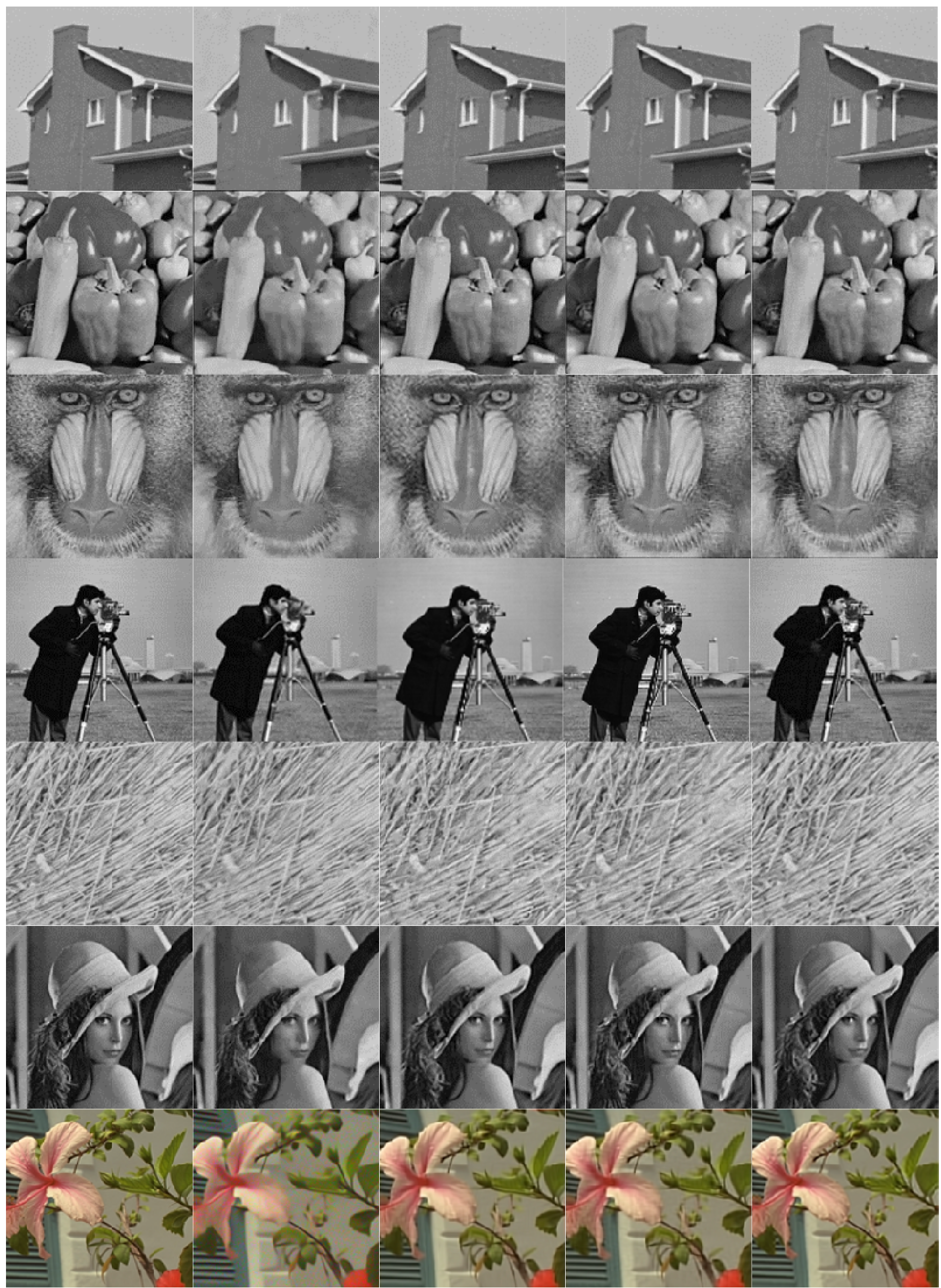

(a) Original HR image

(b) Bicubic Interpolation

(c) Chen [22]

(d) Guo [21]

(e) Proposed method

Fig. 4. Visual analysis and comparison of proposed method with different methods (House, Peppers, Baboon, Cameraman, Straw, Lena and Flower). 


\section{CONCLUSION}

In this paper, a new method is proposed by using learning-based method which focuses on the problem of achieving the goal of SR image. Prior information is used from a high resolution images which is used to generate the good SR image and smooth edges. We introduces dual dictionaries which is the combination of $L R$ and HR dictionaries. To enhance the structure detail between LR and HR images, we develop the nonlinear relationship between LR and HR coefficients by using kernel model. HR patch is generated based on co-sparse with learning subdictionary. Furthermore, propagation filter and self-similarity features have been utilized to enhance the HR patch. At last, the final HR image can be generated with reconstructed all superior HR patches. Simulation results demonstrates that the proposed method achieved better qualitative improvement compared with other competent methods in terms of visual resolution and smooth edges. In addition, it achieves better PSNR and SSIM values.

\section{REFERENCES}

[1] S. C. Park, M. k. Park, and M. G. Kang, "Super-resolution image reconstruction: a technical overview," IEEE Signal Processing Magazine, vol. 20, no. 3, pp. 21-36, May 2003.

[2] Z. Kaibing, D. Tao, X. Gao, X. Li, and Z. Xiong, "Learning multiple linear mappings for efficient single image super-resolution," IEEE Trans. on Image Processing, vol. 24, no. 3, pp. 846-861, March 2015.

[3] J. Allebach, and P. W. Wong, "Edge-directed interpolation," in Proc. IEEE International Conference on Image Processing, vol. 3, pp. 707-710, Lausanne, 1996.

[4] X. Li and M. T. Orchard, "New edge-directed interpolation," IEEE Trans. on Image Processing, vol. 10, no. 10, pp. 1521-1527, Oct 2001.

[5] S. Dai, M. Han, W. Xu, Y. Wu, Y. Gong, and A. K. Katsaggelos, "SoftCuts: A soft edge smoothness prior for color image super-resolution," IEEE Trans. on Image Processing, vol. 18, no. 5, pp 969-981, May 2009.

[6] P. Tsourlos, J. E. Szymanski, and G. N. Tsokas, "A generalized iterative back-projection algorithm for 2-D reconstruction of resistivity data: application to data-sets from archaeological sites," Journal of the Balkan Geophysical Society, vol. 8, no. 2, pp. 37-52, 2005.

[7] M. Irani and S. Peleg, "Improving resolution by image registration," Graph. Models Image Process (CVGIP), vol. 53, no. 5, pp. 231-239, 1991.

[8] Z. Li, G. Han, S. Xiao, and X. Chen, "Map-based single-frame super-resolution image reconstruction for license plate recognition," International Conference on Computational Intelligence and Software Engineering, (CISE), pp. 1-5, Wuhan, China, 2009.

[9] M. C. Hong, M. G. Kang, and A. K. Katsaggelos, "An iterative weighted regularized algorithm for improving the resolution of video sequences," International conference on Image Processing, vol. 2, pp. 474-477, Santa Barbara, CA, 1997.

[10] W. T. Freeman, T. R. Jones, and E. C. Pasztor, "Example-based super-resolution," IEEE Computer Graphics and Applications, vol. 22, no. 2, pp. 56-65, Mar/Apr 2002.

[11] Z. Xiong, D. Xu, X. Sun, and F. Wu, "Example-Based Super-Resolution with Soft Information and Decision," IEEE Trans. on Multimedia, vol. 15, no. 6, pp. 1458-1465, Oct. 2013.

[12] A. Chakrabarti, A. N. Rajagopalan, and R. Chellappa, "Super-resolution of face images using kernel PCA-based prior," IEEE Trans. on Multimedia, vol. 9, no. 4, pp. 888-892, June 2007.

[13] K. Kwang and Y. Kwon, "Example-based learning for single-image super-resolution," in Proc. 30th Annual Symp. Deutsche ArbeitsgemeinSchaft fur Mustererkennung, pp. 456-465, 2008.

[14] A. Milchevski, Z. Ivanovski, and B. Mustafa, "Machine learning based supper-resolution algorithm robust to registration errors," IEEE Signal Processing Education Workshop (DSP/SPE), pp. 326-331, Sedona, AZ, 2011.

[15] Y. Jianchao, J. Wright, T. Huang, and Y. Ma, "Image super-resolution as sparse representation of raw image patches," in Proc. IEEE
Conference on Computer Vision and Pattern Recognition (CVPR), pp. 1-8, Anchorage, AK, 2008

[16] Y. Jianchao, J. Wright, T. S. Huang, and Y. Ma, "Image super-resolution via sparse representation," IEEE Trans. on Image Processing, vol. 19, no. 11, pp. 2861-2873, Nov. 2010.

[17] Y. Guoshen and S. Mallat, "Sparse super-resolution with space matching pursuits," SPARS'09-Signal Processing with Adaptive Sparse Structured Representations, pp. 1-6, 2009.

[18] R. Zeyde, M. Elad, and M. Protter, "On single image scale-up using sparse-representations," in Proc. International Conference of Curves and Surfaces, pp. 711-730, Avignon, France, June 2012.

[19] S. Yicheng, G. Guohua, S. Xiubao, L. Yuan, and Y. Chengzhang, "Single image super-resolution using compressive sensing with a redundant dictionary," IEEE Photonics Journal, vol. 7, no. 2, pp. 1-11, 2015.

[20] D. Weisheng, Z. Lei, G. Shi, and W, Xiaolin, "Image deblurring and super-resolution by adaptive sparse domain selection and adaptive regularization," IEEE Trans. on Image Processing, vol. 20, no. 7, pp. 1838-1857, 2011.

[21] S. Gou, S. Liu, Y. Wu, and L. Jiao, "Image super-resolution based on the pairwise dictionary selected learning and improved bilateral regularisation," IET Image Processing, vol. 10, no. 2, pp. 101-112, 2016.

[22] H. Chen, J. Xue, S. Zhang, Y. Lu, C. Guo, "Image super-resolution based on adaptive cosparse regularisation," Electronics Letters, vol. 50, no. 24, pp. 1834-1836, 2014.

[23] Z. Xiaoqiang, and J. Yunxia, "Image super-resolution reconstruction algorithm based on clustering," in Proc. the 27th Chinese Control and Decision Conference (2015 CCDC), pp. 6144-6148, Qingdao, 2015.

[24] K. Fukunaga, Introduction to Statistical Pattern Recognition, 2nd ed. New York: Academic, 1991.

[25] L. Zhang, R. Lukac, X. Wu, and Z. David, "PCA-based spatially adaptive denoising of CFA images for single-sensor digital cameras," IEEE Trans. on Image Processing, vol. 18, no. 4, pp. 797-812, 2009.

[26] W. Yang, T. Yuan, W. Wang, F. Zhou, and Q. Liao, "Single-Image Super-Resolution by Subdictionary Coding and Kernel Regression," IEEE Trans. on Systems, Man, and Cybernetics: Systems, no. 99 , pp.1-11, 2016.

[27] S. Nam, M.E Davies, M. Elad, and R. Gribonval, "Cosparse analysis modeling - Uniqueness and algorithms," IEEE International Conference on Acoustics, Speech and Signal Processing (ICASSP), pp. 5804-5807, Prague, May 2011.

[28] R. Giryes, Y. Plan, and R. Vershynin, "On the Effective Measure of dimension in the analysis cosparse model," IEEE Trans. on Information Theory, vol. 61, no. 10, pp. 5745-5753, Oct. 2015.

[29] J. H. R. Chang, and Y. C. F. Wang, "Propagated image filtering," in Proc. IEEE Conference on Computer Vision and Pattern Recognition (CVPR), pp. 10-18, Boston, MA, 2015.

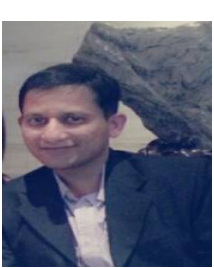

Muhammad Sameer Sheikh was born in Karachi Pakistan, he received his bachelor degree in electronic Engineering FROM Hamdard University, Karachi, Pakistan in 2007. He received his master degree in telecommunication engineering from Hamdard University Karachi, Pakistan in 2010. He is currently a $\mathrm{PhD}$ candidate at college of Electronics and Information Engineering, Nanjing University of Aeronautics and Astronautics. Nanjing, China his main research interest focused on the area of computational electromagnetics, radar signal processing, image processing and digital signal processing.

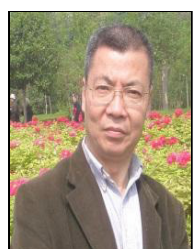

Qunsheng Cao received his Ph.D. in electrical engineering from The Hong Kong Polytechnic University in 2001. From 2001 to 2005 he worked as a research associate in the Department of Electrical Engineering, University of Illinois at Urbana-Champaign and at the Army High Performance Computing Research Center (AHPCRC), University of Minnesota. In 2006, Dr. Cao joined the Nanjing University of Aeronautics and Astronautics (NUAA), China, as a professor of electrical engineering. Dr. Cao's current research interests are in computational electromagnetics, antenna and microwave technology and the radar signal processing. Dr. Cao has published more than 120 academic papers in refereed journals and conference proceedings. 


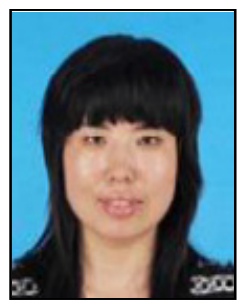

Caiyun Wang was born in Shanxi, China, on September 30, 1975. She graduated in 1996 with a B.S. degree and in 1999 with a M.S. degree. She received the $\mathrm{Ph} . \mathrm{D}$. degree in signal and information processing from Beihang University, Beijing, China, in 2008.
She is currently an associate professor with the College of Astronautics, Nanjing University of Aeronautics and Astronautics (NUAA). Her major research interests are in the fields of radar automatic target recognition (RATA), radar signal processing, image processing, digital signal processing and compress sensing. 